\title{
Wilson lines on noncommutative tori
}

\author{
Anton Yu. Alekseev ${ }^{\dagger}$, Andrei G. Bytsko* \\ $\dagger^{\dagger}$ Institute for Theoretical Physics, Uppsala University, Box 803, S-75108, Uppsala, Sweden \\ alekseev@teorfys.uu.se \\ * Steklov Mathematics Institute, Fontanka 27, St. Petersburg 191011, Russia \\ bytsko@pdmi.ras.ru \\ hep-th/0002101 \\ (February, 2000)
}

\begin{abstract}
We introduce the notion of a monodromy for gauge fields with vanishing curvature on the noncommutative torus. Similar to the ordinary gauge theory, traces of the monodromies define noncommutative Wilson lines. Our main result is that these Wilson lines are invariant under the Seiberg-Witten map changing the deformation parameter of the noncommutative torus.
\end{abstract}

PACS-98: 11.15.-q, 11.10.Kk, 02.90.+p

\section{INTRODUCTION}

Gauge theories on noncommutative spaces [1] arise as low energy effective theories on $D$-brane world volumes in the presence of the background $B$-field in the theory of open strings [2, 3,, . The simplest noncommutative space where a gauge theory can be constructed is the space $\mathbf{R}_{\theta}^{d}$ with coordinates $x^{i}$ obeying the Heisenberg commutation relations,

$$
\left[x^{i}, x^{j}\right]=i \theta^{i j},
$$

where $\theta^{i j}=-\theta^{j i}$ is a constant anti-symmetric matrix. In string theory, $\theta$ is given by the following formula [3, 4 ]

$$
\theta=-\left(2 \pi \alpha^{\prime}\right)^{2}\left(g+2 \pi \alpha^{\prime} B\right)^{-1} B\left(g-2 \pi \alpha^{\prime} B\right)^{-1},
$$

where $\alpha^{\prime}$ is the inverse to the string tension, $g$ is the metric and $B$ is the $B$-field on the brane world-volume. Functions on the space $\mathbf{R}_{\theta}^{d}$ can be identified with ordinary functions on $\mathbf{R}^{d}$ with the noncommutative product given by the Moyal formula,

$$
(u * v)(x)=\left(e^{\frac{i}{2} \theta^{i j} \partial_{i}^{x} \partial_{j}^{y}} u(x) v(y)\right)_{y=x} .
$$

Along with the space $\mathbf{R}_{\theta}^{d}$ it is natural to consider its compactification $T_{\theta}^{d}$. We can choose the compactification radii $R_{i}$ such that the functions on $T_{\theta}^{d}$ are invariant with respect to the shifts,

$$
f\left(x^{1}+2 \pi n_{1} R^{1}, \ldots, x^{d}+2 \pi n_{d} R^{d}\right)=f\left(x^{1}, \ldots, x^{d}\right),
$$

where $n_{i}$ are arbitrary integers. Since $\theta^{i j}$ is a constant tensor, the Moyal product is invariant with respect to such shifts. Therefore, the $*$-product restricts to periodic functions.
An important ingredient in the ordinary gauge theory is the notion of a Wilson line,

$$
W_{\gamma}(A)=\operatorname{Tr} P \exp \left(i \int_{\gamma} A\right)
$$

where $A$ is a gauge field and $\gamma$ is a closed contour. The expression $W_{\gamma}(A)$ is gauge invariant and, hence, defines an observable in the gauge theory. One reason why $W_{\gamma}(A)$ is an important object is given by the 't Hooft's criterion of confinement [5]. Another reason is as follows. Suppose that the curvature of $A$,

$$
F_{i j}=\partial_{i} A_{j}-\partial_{j} A_{i}+i\left[A_{j}, A_{i}\right],
$$

vanishes. Locally, the condition $F=0$ implies that $A$ is a pure gauge. Globally, flat connections (gauge fields with vanishing curvature) may have moduli. One can distinguish a nontrivial gauge field with $F=0$ from a pure gauge by looking at the Wilson lines along noncontractible contours. The standard application of this principle is the Aharonov-Bohm effect where the phase of the Wilson line for electromagnetic vector potential influences the interference pattern.

It is the goal of this paper to study the notion of a Wilson line in a gauge theory on the noncommutative torus $T_{\theta}^{d}$. In contrast to the previous suggestions [6,7], we only consider the case of flat connections. In our view, there is a conceptual difficulty in the case of $F_{i j} \neq 0$ : the definition of a Wilson line contains a contour $\gamma$. It might be a nontrivial task to find noncommutative subspaces of a noncommutative space. Technically speaking, the requirement is that the algebra of functions vanishing on the subspace be an ideal in the noncommutative algebra of functions. For instance, if $\theta$ is nondegenerate and generic, the noncommutative torus $T_{\theta}^{d}$ has no subspaces different from $T_{\theta}^{d}$ itself. In particular, there is not a single 'noncommutative contour' on $T_{\theta}^{d}$. In the case of 
$F_{i j}=0$, the Wilson line only depends on the homotopy class of the contour $\gamma$. We propose a natural gauge invariant object which generalizes $W_{\gamma}(A)$ in the case of flat noncommutative gauge fields.

It has been shown in [4] that one can construct a transformation (the Seiberg-Witten map) which identifies the noncommutative gauge fields on $\mathbf{R}_{\theta}^{d}$ (and on $T_{\theta}^{d}$ ) for different values of $\theta$. The Seiberg-Witten map on general noncommutative spaces was recently studied in [8]. We show that our noncommutative Wilson line is invariant with respect to the Seiberg-Witten map. In other words, we prove that the Seiberg-Witten map for flat connections is isomonodromic.

It would be very interesting to see whether the noncommutative Wilson lines have some natural interpretation in the $D$-brane Physics. The following elementary example can be used as a starting point. The energy spectrum of a non-relativistic particle of mass $m$ and charge $q$ confined to a circle of length $L$ in the constant vector potential $A$ has the form,

$$
E_{n}=\frac{1}{2 m} \frac{4 \pi^{2} \hbar^{2}}{L^{2}}(n+i \ln (M))^{2}
$$

where

$$
M=\exp \left(i \frac{q A L}{2 \pi \hbar}\right)
$$

is the monodromy of the Wilson line winding around the circle. In the case of noncommutative torus, the charged particle is replaced by the end-point of an open string. It is plausible that the noncommutative Wilson line shifts the spectrum of an open string in a similar fashion.

\section{NONCOMMUTATIVE WILSON LINES}

In ordinary gauge theory, there are two ways to define a Wilson line for a gauge field $A$ with vanishing curvature $F$. For the purpose of this paper we restrict our attention to the Wilson lines on the torus $T^{d}$. Since a gauge field with vanishing curvature is locally a pure gauge, one can solve the equation,

$$
\partial_{i} g(x)=i A_{i}(x) g(x),
$$

where $g$ is not necessarily periodic. Different solutions of (田) can be obtained from each other by multiplication by a constant from the right, $g(x) \rightarrow g(x) h$. Under gauge transformations solutions of equation (4) get multiplied from the left, $g(x) \rightarrow h(x) g(x)$.

One can now introduce left and right monodromy matrices, $M_{i}=g^{-1} g_{i}$ and $\tilde{M}_{i}=g_{i} g^{-1}$, where $g \equiv$ $g\left(x^{1}, \ldots, x^{d}\right)$ and $g_{i} \equiv g\left(x^{1}, \ldots, x^{i}+2 \pi R^{i}, \ldots, x^{d}\right)$ are solutions of (化). It is easy to see that the monodromies $M_{i}$ are gauge-invariant and do not depend on the point $x$. If one replaces the solution $g(x)$ by some other solution of equation (14), $g(x) h$, the monodromy $M_{i}$ gets conjugated, $M_{i} \rightarrow h^{-1} M_{i} h$. Therefore, the trace of $M_{i}$ is a gauge invariant object which is independent of the choice of a solution of (4) and can be used as the definition of the Wilson line, $W_{i}=\operatorname{Tr} M_{i}$.

The monodromies $\tilde{M}_{i}$ are the usual ordered exponentials,

$$
\tilde{M}_{i}=P \exp \left(i \int_{x^{i}}^{x^{i}+2 \pi R^{i}} A\right) .
$$

They are independent of the choice of the solution $g(x)$ but have explicit dependence on the point $x$ and transform covariantly under the gauge transformations, $\tilde{M}_{i} \rightarrow$ $h(x) \tilde{M}_{i} h(x)^{-1}$. Again, taking a trace yields a gauge invariant object independent of all choices which coincides with $W_{i}$.

In the noncommutative gauge theory, only the prescription which uses monodromies $M_{i}$ survives. It is still possible to construct objects similar to $\tilde{M}_{i}$ (see e.g. [7]) which transform covariantly under the gauge transformations, $\tilde{M}_{i} \rightarrow h(x) * \tilde{M}_{i} * h(x)^{-1}$. However, the matrix trace fails to be cyclic under the Moyal product, that is $\operatorname{Tr}(u * v) \neq \operatorname{Tr}(v * u)$. This implies that $\operatorname{Tr} \tilde{M}_{i}$ is not gauge-invariant.

In what follows we present the definition of a Wilson line in noncommutative gauge theory which uses the monodromy $M_{i}$. In the noncommutative case, the curvature of the gauge field is defined using the $*$-product (see e.g. 画),

$$
F_{i j}=\partial_{i} A_{j}-\partial_{j} A_{i}-i A_{i} * A_{j}+i A_{j} * A_{i} .
$$

The infinitesimal gauge transformations are of the form,

$$
\delta_{\lambda} A_{i}=\partial_{i} \lambda+i \lambda * A_{i}-i A_{i} * \lambda .
$$

Let us mention that not every compact Lie group can be used as a gauge group on $T_{\theta}^{d}$. For instance, $G=U(N)$ works for any $\theta$ whereas $G=S U(N)$ in general does not give rise to a gauge group on the noncommutative torus. More precisely, the $*$-product $g * h$ of two unitary matrices is always unitary, but in general $\operatorname{det}(g * h) \neq$ $\operatorname{det}(g) * \operatorname{det}(h)$.

Equations (5) and (6) are obtained from the standard formulas in ordinary (commutative) gauge theory by introducing $*$-products instead of the ordinary products. It is slightly more complicated to find a counterpart of the general formula for gauge transformations,

$$
A_{i}^{h}=-i \partial_{i} h * h_{*}^{-1}+h * A_{i} * h_{*}^{-1} .
$$


Here $h(x)$ is an element of the gauge group, and $h_{*}^{-1}$ is the inverse of $h$ with respect to the $*$-product, $h * h_{*}^{-1}=$ $h_{*}^{-1} * h=1$. Note that for $\theta \neq 0$ the element $h_{*}^{-1}$ differs from the ordinary inverse $h^{-1}$. More explicitly,

$$
h_{*}^{-1}=h^{-1}+\frac{i}{2} \theta^{k l} h^{-1}\left(\partial_{k} h\right) h^{-1}\left(\partial_{l} h\right) h^{-1}+\mathcal{O}\left(\theta^{2}\right) .
$$

We now turn to the case of a noncommutative gauge field $A$ with vanishing curvature $F=0$. Then, one may expect that at least locally $A$ is a pure gauge. That is, there is an element of the gauge group $g$ such that,

$$
\partial_{i} g=i A_{i} * g .
$$

At this point it is convenient to view gauge fields on $T_{\theta}^{d}$ as periodic gauge fields on $\mathbf{R}_{\theta}^{d}$. Then, we expect that equation (8) admits global solutions. For now we assume that such solutions exist and return to this issue in the next section.

Our next task is to show that the ratio $m=g_{*}^{-1} * g^{\prime}$ of two solutions $g$ and $g^{\prime}$ of equation (8) is a constant. Indeed,

$$
\begin{aligned}
& \partial_{i} m=-g_{*}^{-1} * \partial_{i} g * g_{*}^{-1} * g^{\prime}+g_{*}^{-1} * \partial_{i} g^{\prime} \\
& =-i g_{*}^{-1} *\left(A_{i}-A_{i}\right) * g^{\prime}=0 .
\end{aligned}
$$

Let us mention that in the derivation of this equation we have used that the $*$-product on $\mathbf{R}_{\theta}^{d}$ satisfies the Leibniz rule, $\partial_{i}(f * g)=\partial_{i} f * g+f * \partial_{i} g$. In general, this property does not hold on noncommutative spaces.

If $g\left(x^{1}, \ldots, x^{d}\right)$ is a solution of (8) then so is $g_{i}\left(x^{1}, \ldots, x^{d}\right)=g\left(x^{1}, \ldots, x^{i}+2 \pi R^{i}, \ldots, x^{d}\right)$. By equation (9), the ratio $M_{i}=g_{*}^{-1} * g_{i}$ is independent of the point on $T_{\theta}^{d}$. We call the group elements $M_{i}$ noncommutative monodromies of the equation (8). As usual, the value of the monodromy depends on the choice of the solution $g(x)$. For some other solution, $g^{\prime}(x)=g(x) * h=$ $g(x) h$ one gets, $M_{i}^{\prime}=h^{-1} g_{*}^{-1} * g_{i} h=h^{-1} M_{i} h$. Here we used that the $*$-product with a constant coincides with the ordinary product. Finally, we can define the Wilson lines which are independent of the choice of the solution $g(x)$,

$$
W_{i}(A)=\operatorname{Tr} M_{i}=\operatorname{Tr}\left(g_{*}^{-1} * g_{i}\right) .
$$

Let us stress, that, since $M_{i}$ are coordinate independent, we use the ordinary matrix trace here. Similar to their commutative counterparts (3), the Wilson lines $W_{i}(A)$ are gauge invariant,

$$
W_{i}\left(A^{h}\right)=\operatorname{Tr}\left(g_{*}^{-1} * h_{*}^{-1} * h * g_{i}\right)=W_{i}(A),
$$

where $g(x) \rightarrow h(x) * g(x)$ is the composition of two gauge transformations.
We put forward the definition (10) of noncommutative Wilson lines $W_{i}(A)$. In the next Section we apply the Seiberg-Witten map to the noncommutative gauge theory at hand and show that our Wilson lines $W_{i}(A)$ are invariant with respect to this transformation.

Let us remark that although the considerations of this Section are restricted to the case of gauge fields with vanishing curvature, they may be viewed as the general definition of a noncommutative Wilson line. Indeed, in the commutative case the Wilson lines are assigned to closed contours. When restricted to a 1-dimensional contour, any gauge field has vanishing curvature and one can use the monodromy $M$ instead of the ordered exponential $\tilde{M}$ to construct the Wilson line. In the noncommutative context it is more difficult to construct submanifolds. In particular, as we pointed out in Introduction, the noncommutative tori with generic $\theta$ have no nontrivial submanifolds. However, if one can find a subtorus $\Gamma$ of the noncommutative torus $T_{\theta}^{d}$ such that the restriction of the gauge field $A$ to $\Gamma$ has vanishing curvature, one can define the Wilson lines of $A$ along $\Gamma$. If $\Gamma$ is 1-dimensional, it defines one Wilson line observable, similar to commutative gauge theory.

\section{THE SEIBERG-WITTEN MAP}

It was established in [1] that the gauge theories on noncommutative tori $T_{\theta}^{d}$ with different values of the deformation parameter $\theta$ are equivalent to each other. More explicitly, let $\theta$ and $\theta+\delta \theta$ be two infinitesimally close values of the deformation parameter. Then, there exists the Seiberg-Witten map $A \rightarrow \hat{A}(A)$ and $\lambda \rightarrow \hat{\lambda}(\lambda, A)$ of the gauge fields and infinitesimal gauge parameters on $\mathbf{R}_{\theta}^{d}$ to those on $\mathbf{R}_{\theta+\delta \theta}^{d}$ such that

$$
\hat{A}\left(A+\delta_{\lambda} A\right)=\hat{A}(A)+\delta_{\hat{\lambda}(\lambda, A)} \hat{A}(A) .
$$

This map is given by explicit formulas (see equation (3.8) in (4). In this paper we only need the form of the SeibergWitten transformation for $A$ with $F=0$,

$$
\delta_{\theta} A_{i}=-\frac{1}{4} \delta \theta^{k l}\left(A_{k} * \partial_{l} A_{i}+\partial_{l} A_{i} * A_{k}\right) .
$$

We show in the Appendix that under the Seiberg-Witten map solutions of the equation (8) transform according to formula,

$$
\delta_{\theta} g=\frac{1}{4 i} \delta \theta^{k l} A_{k} * A_{l} * g .
$$

It is convenient to introduce a special notation for the 'gauge parameter',

$$
\lambda(\delta \theta, A)=-\frac{1}{4} \delta \theta^{k l} A_{k} * A_{l} .
$$


Note, however, that $\delta_{\theta} A_{i}$ is not a gauge transformation. More exactly,

$$
\delta_{\lambda(\delta \theta, A)} A_{i}=-\frac{1}{4} \delta \theta^{k l}\left(A_{k} * \partial_{l} A_{i}-\partial_{l} A_{i} * A_{k}\right) .
$$

The reason is that in contrast to partial derivatives $\partial_{i}$, the transformation $\delta_{\theta}$ violates the Leibniz rule for the Moyal product. Indeed, the exponential form of (2) implies,

$$
\delta_{\theta}(u * v)=\left(\delta_{\theta} u\right) * v+u *\left(\delta_{\theta} v\right)+\frac{i}{2} \delta \theta^{i j} \partial_{i} u * \partial_{j} v .
$$

In particular, putting together equations (12)-(15) we obtain,

$$
\delta_{\theta}\left(g_{*}^{-1}\right)=g_{*}^{-1} * \delta_{\theta} g * g_{*}^{-1} .
$$

Surprisingly, there is no minus sign on the right hand side. This is due to the contribution of the last term in (15).

We are now prepared to address the question of variation of the Wilson lines under the Seiberg-Witten map. Let us apply the transformation $\delta_{\theta}$ to equation $g_{i}(x)=$ $g(x) M_{i}$. The result reads,

$$
i \lambda(\delta \theta, A) * g_{i}=i \lambda(\delta \theta, A) * g M_{i}+g \delta_{\theta} M_{i} .
$$

Thus, we conclude that $\delta_{\theta} M_{i}=0$ and monodromies $M_{i}$ are $\theta$-independent, and so are the Wilson lines $W_{i}(A)$.

Finally, we return to the question of existence of solution $g(x)$ of the equation (8). Clearly, such solutions exist for $\theta=0$. We can use these solutions as initial conditions in the pair of differential equations (12) and (13). Choosing a path between $\theta_{0}=0$ and some other value $\theta_{1}$, one can, at least formally, obtain solutions of equation (8) for $\theta=\theta_{1}$ by solving equations (12) and (13). In general, these solutions depend on the path between $\theta_{0}$ and $\theta_{1}$. In other words, two infinitesimal transformations $\delta_{\theta}^{1}$ and $\delta_{\theta}^{2}$ may have a nonvanishing commutator. This commutator applied to gauge fields $A$ was first computed in [9]. We obtain an elegant formula for the variation of $g$,

$$
\left[\delta_{\theta}^{1}, \delta_{\theta}^{2}\right] g=\frac{1}{16} \delta \theta_{1}^{k l} \delta \theta_{2}^{m n}\left(\partial_{l} A_{n} * \partial_{m} A_{k}-\partial_{m} A_{k} * \partial_{l} A_{n}\right) .
$$

There is no obvious reason for the right hand side to vanish even in the abelian case for $\theta \neq 0$.

\section{APPENDIX}

Our goal is to show that transformation (13) is consistent with formula (8) provided that eq. (12) holds. Applying the partial derivative $\partial_{i}$ to equation(13) and then using twice that $F=0$, we obtain

$$
\begin{aligned}
& \delta_{\theta}\left(\partial_{i} g\right)= \\
& =\frac{1}{4 i} \delta \theta^{k l}\left(\partial_{i} A_{k} * A_{l}+A_{k} * \partial_{i} A_{l}+i A_{k} * A_{l} * A_{i}\right) * g \\
& =\frac{1}{4 i} \delta \theta^{k l}\left(\partial_{k} A_{i} * A_{l}+A_{k} * \partial_{l} A_{i}+i A_{i} * A_{k} * A_{l}\right) * g .
\end{aligned}
$$

Taking into account the antisymmetry of $\theta^{i j}$, we can rewrite this expression as follows:

$$
\begin{aligned}
& \delta_{\theta}\left(\partial_{i} g\right)=\frac{1}{4 i} \delta \theta^{k l}\left(2 \partial_{k} A_{i} * A_{l}+\partial_{l} A_{i} * A_{k}\right. \\
& \left.\quad+A_{k} * \partial_{l} A_{i}+i A_{i} * A_{k} * A_{l}\right) * g \\
& =-\frac{1}{2} \delta \theta^{k l} \partial_{k} A_{i} * \partial_{l} g+i\left(\delta_{\theta} A_{i}\right) * g+i A_{i} * \delta_{\theta} g \\
& =i \delta_{\theta}\left(A_{i} * g\right) .
\end{aligned}
$$

Here we used formulas (12) and (13) in the third line, and (8) and (15) in the last line.

Acknowledgments: We would like to thank K. Okuyama, V. Roubtsov and V. Schomerus for useful discussions. A.A. thanks the organizers and the participants of the conference on Noncommutative Gauge Theory (Leiden, November 1999) for inspiring discussions. The visit of A.B. to the Institute for Theoretical Physics, Uppsala University was supported by the grant INTAS 96-196 and by the grant VISBY-380 of the Swedish Institute.

[1] A. Connes, Noncommutative Geometry (Academic Press, 1994); A. Connes, M.R. Douglas, A. Schwarz, JHEP 9802:003 (1998); A. Schwarz, Nucl. Phys. B534 (1998) 720-738.

[2] M.R. Douglas, C. Hull, JHEP 9802:008 (1998); Y.K.E. Cheung, M. Krogh, Nucl. Phys. B528 (1998) 185-196; G. Landi, F. Lizzi, R.J. Szabo, Commun. Math. Phys. 206 (1999) 603-637; C.-S. Chu, P.-M. Ho, Nucl. Phys. B550 (1999) 151-168; F. Ardalan, H. Arfaei, M.M. SheikhJabbari, JHEP 9902:016 (1999); L. Cornalba, hepth/9909081 (1999).

[3] V. Schomerus, JHEP 9906:030 (1999).

[4] N. Seiberg, E. Witten, JHEP 9909:032 (1999).

[5] G. 't Hooft, Nucl. Phys. B190 (1981) 455-478.

[6] N. Ishibashi, S. Iso, H. Kawai, Y. Kitazawa, hep-th/9910004 (1999); O. Andreev, H. Dorn, Phys. Lett. B476 (2000) 402410.

[7] K. Okuyama, JHEP 0003:016 (2000).

[8] B. Jurco, P. Schupp, hep-th/0001032 (2000); J. Madore, S. Schraml, P. Schupp, J. Wess, hep-th/0001203 (2000).

[9] T. Asakawa, I. Kishimoto, JHEP 9911:024 (1999). 